\title{
HIGH-BRIGHTNESS BEAM DIAGNOSTICS FOR THE APS LINAC
}

\author{
A. H. Lumpkin, B. X. Yang, W. J. Berg, J. W. Lewellen and S. V. Milton \\ Advanced Photon Source, Argonne National Laboratory, Argonne, IL 60439 \\ The Advanced Photon Source (APS) injector includes an

\section{EXPERIMENTAL BACKGROÛND}

Abstract $S$-band linac with the capability to accelerate beams to $650 \mathrm{MeV}$. The linac has recently been upgraded with the installation of an if thermionic gun in addition to the standard DC thermionic gun. The rf gun is predicted to have lower emittance ( $5 \pi \mathrm{mm} \mathrm{mrad}$ ) and may be used to support the APS self-amplified spontaneous emission (SASE) experiments. The critical characterization of this gun's beam has begun with a beam diagnostics station at the end of the linac that can address beam transverse size, emittance, and bunch length (peak current). This station uses both an optical transition radiation (OTR) screen at $45^{\circ}$ to the beam direction and a Ce-doped YAG single crystal normal to the beam with a $45^{\circ}$ mirror behind it. The visible light images are detected by a Vicon CCD camera and a Hamamatsu C5680 synchroscan streak camera. Spatial resolution of about $30 \mu \mathrm{m}(\sigma)$ and temporal resolution of $1 \mathrm{ps}(\sigma)$ have been demonstrated. Examples of if gun beam characterization at $220 \mathrm{MeV}$ are reported.

\section{INTRODUCTION}

The ongoing interest in high-brightness particle beams has been "stimulated" by the potential applications to self-amplified spontaneous emission (SASE) free-electron laser experiments [1,2]. At the Advanced Photon Source (APS) the injector linac has recently been upgraded with the installation of an if thermionic gun $[3,4]$ and a laserdriven photocathode (PC) if gun [5]. The if gun is predicted to have lower emittance $(5 \pi \mathrm{mm} \mathrm{mrad})$ and sufficient peak currents $(\sim 100 \mathrm{~A})$ to be used in the APS SASE experiments [6]. The PC rf gun is expected to have even better brightness [5]. The capability to characterize such beams includes a beam diagnostic station with improved spatial and temporal resolution that can address beam transverse size, emittance, and bunch length (peak current). The station uses both optical transition radiation (OTR) and a Ce-doped YAG single crystal normal to the beam with a mirror behind it. The visible light images were detected by a charge-coupled device (CCD) camera and a dual-sweep streak camera. Spatial resolution of about $30 \mu \mathrm{m}(\sigma)$ and temporal resolution of about $1 \mathrm{ps}(\sigma)$ have been demonstrated in earlier studies $[7,8]$. Examples of beam measurements for the if gun are reported. *Work supported by the U.S. Department of Energy, Office of Basic
Energv Sciences. under Contract No. W-31-109-ENG-38.
The APS facility's injector system uses a $250-\mathrm{MeV}$ Sband electron linac and an in-line $450-\mathrm{MeV}$ S-band positron linac. The original electron gun was a conventional, gated DC thermionic gun. The diagnostics station was initially commissioned using this gun's beam [7]. More recently an tf thermionic gun was installed that is designed to generate low-emittance beams $(<5 \pi \mathrm{mm}$ mrad). It is configured with an alpha magnet that allows beam injection just after the first linac accelerating section [3,4]. Both in-line linacs can be phased to produce 50 - to $650-\mathrm{MeV}$ electron beams when the positron converter target is retracted.

The predicted lower normalized emittance of beams from the if thermionic gun and PC If gun versus the DC gun results in correspondingly smaller beam spot sizes. We have addressed this at selected diagnostics stations by supplementing or replacing the standard Chromox screen, with its approximate $200-\mu \mathrm{m}$ spatial resolution and 300 ms decay time, with the OTR and Ce-doped YAG screens. For the station at the end of the linac the OTR screen was a molybdenum mirror from Melles Griot and oriented so that its surface was at $45^{\circ}$ to the beam direction. The doped YAG crystal of $0.5 \mathrm{~mm}$ thickness (obtained from Startec) was mounted with its surface normal to the beam direction with a Zerodur mirror at $45^{\circ}$ to the beam just behind it.

An alignment laser was injected on-axis into the bore of the linac just after the first accelerating structure and used to assess the relative orientation of the two mirrors involved at the station. The laser was reflected into the optical transport line and then used to simulate the path of the visible light images. In the case of OTR, the direction of the path is particularly critical since OTR is emitted around the angle of specular reflection. The visible light was transported out of the linac tunnel to an optics table via two $150-\mathrm{mm}$-diameter achromat lenses and two mirrors. For these experiments the OTR or YAG:Ce light could be viewed by a Vicon CCD camera and/or a Hamamatsu C5680 dual-sweep streak camera. The synchroscan unit was phase-locked to $119.0 \mathrm{MHz}$, the $24^{\text {th }}$ subharmonic of the $2856-\mathrm{MHz}$ linac frequency. A lowjitter countdown circuit has been built using Motorola ECLIN PS Logic to generate the $24^{\text {th }}$ subharmonics with sub-ps jitter [9]. The synchroscan unit was critical to the bunch length measurements due to the lower charge/micropulse in early experiments. The model M5677 slow-sweep unit was used in the measurement of the YAG-Ce response time, which was previously 


\section{DISCLAIMER}

This report was prepared as an account of work sponsored by an agency of the United States Government. Neither the United States Government nor any agency thereof, nor any of their employees, make any warranty, express or implied, or assumes any legal liability or responsibility for the accuracy, completeness, or usefuiness of any information, apparatus, product, or process disclosed, or represents that its use would not infringe privately owned rights. Reference herein to any specific commercial product, process, or service by trade name, trademark, manufacturer, or otherwise does not necessarily constitute or imply its endorsement, recommendation, or favoring by the United States Government or any agency thereof. The views and opinions of authors expressed herein do not necessarily state or reflect those of the United States Government or any agency thereof. 


\section{DISCLAIMER}

Portions of this document may be illegible in electronic image products. Images are produced from the best available original document. 
reported [10] as about 80 ns (FWHM). Although beam energies as high as $540 \mathrm{MeV}$ have been used, one set of data is at $220 \mathrm{MeV}$, the energy that would be used for SASE experiments at $517 \mathrm{~nm}$.

\section{EXPERIMENTAL RESULTS}

Preliminary results are reported in this section on rf gun beam transverse size, emittance, and bunch length. Additionally, the measurement of the YAG:Ce crystal response time is reported.

\subsection{Beam Transverse Size}

Recently measurements at $220 \mathrm{MeV}$ were performed on the beam at the end of the linac. Beam images were obtained with both the YAG:Ce crystal and the OTR converter. Figure 1 shows an example of the beam vertical profile using OTR. The MAC-TA algorithm calculated a spot size of $\sim 170 \mu \mathrm{m}$ (FWHM) that corresponds to about $72 \mu \mathrm{m}(\sigma)$, assuming a Gaussianshaped profile. The aspect ratio $(\mathrm{H}: \mathrm{V})$ was about $4: 1$ for this beam focus.

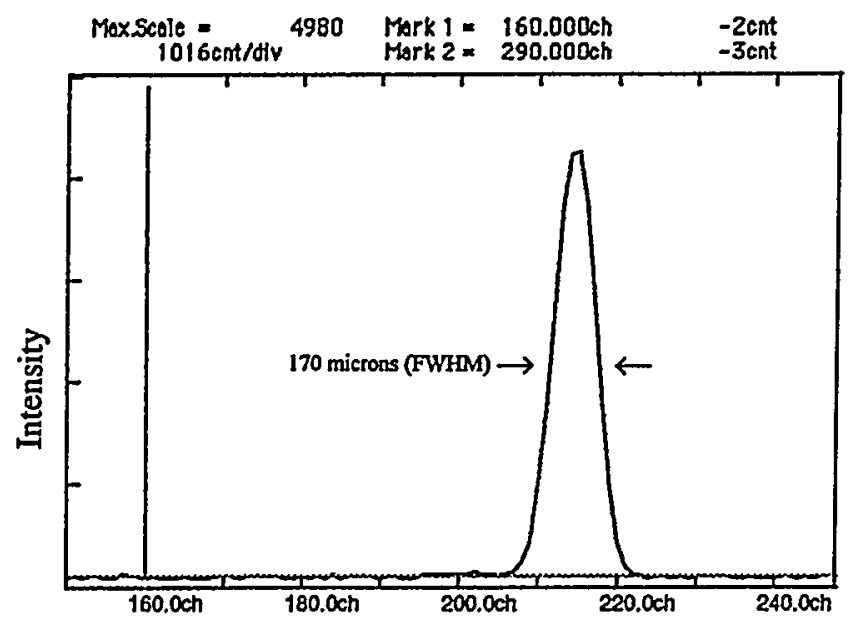

\section{Vertical Position (ch)}

Figure 1: An example of the rf thermionic gun's vertical beam profile: $170 \mu \mathrm{m}$ (FWHM).

\subsection{Preliminary Emittance}

Having obtained reasonable transport and focus of the beam, an evaluation of the beam emittance was performed by the standard tracking of beam size with the quadrupole field strength of an upstream magnet. In this screen position there were two quadrupoles that were available to be adjusted. A script developed by $M$. Borland was adapted that automatically stepped the fields and logged the measured beam sizes via the EPICS process variables [11]. As shown in Fig. 2, the horizontal beam size can be minimized by selecting a combination of fields from the linac-to-PAR magnets LTPQ9 and LTPQ10. The Max Video-20 digitized data were fit to Gaussian profiles in this case. An example of emittance data is shown in Fig. 3. The data are consistent with normalized emittances, $\varepsilon_{\mathrm{x}}=8.8 \pi \mathrm{mm} \mathrm{mrad}$ and $\varepsilon_{\mathrm{y}}=9.5$ $\pi \mathrm{mm} \mathrm{mrad}$ for a $25-\mathrm{mA}$ macropulse current. Errors are estimated as $20-30 \%$. Simulations of this particular if gun setup with elegant [12] have reproduced the emittance asymmetry between the two planes. It is noted that the measured and computed emittance ratios are similar although the measured values are $60 \%$ higher. These measurements were made without an energy filter in the alpha magnet. As a result, the beam contains a trailing, low-energy component that is expected to significantly increase the projected (but not slice) emittance. Although this low-energy "tail" contains only a small fraction of the total charge (approximately $10 \%$ of the total bunch charge by the time the bunch reaches the end of the linac), it is the greatest contribution to the projected (longitudinal whole-beam) emittance. Considerably lower measured emittances are expected once the alpha magnet energy filter has been installed.

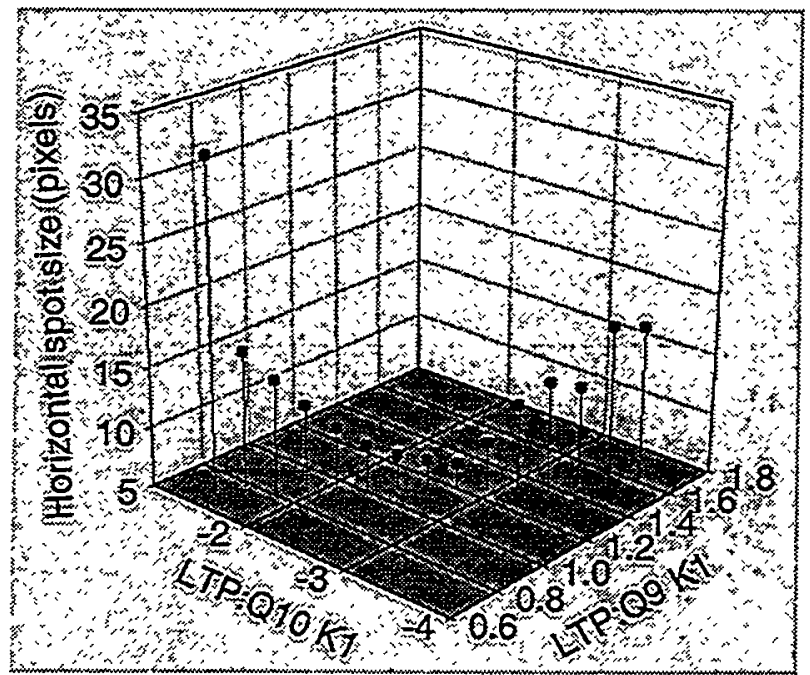

Figure 2: Variations of the observed horizontal beam size with the two upstream quadrupoles Q9 and Q10.

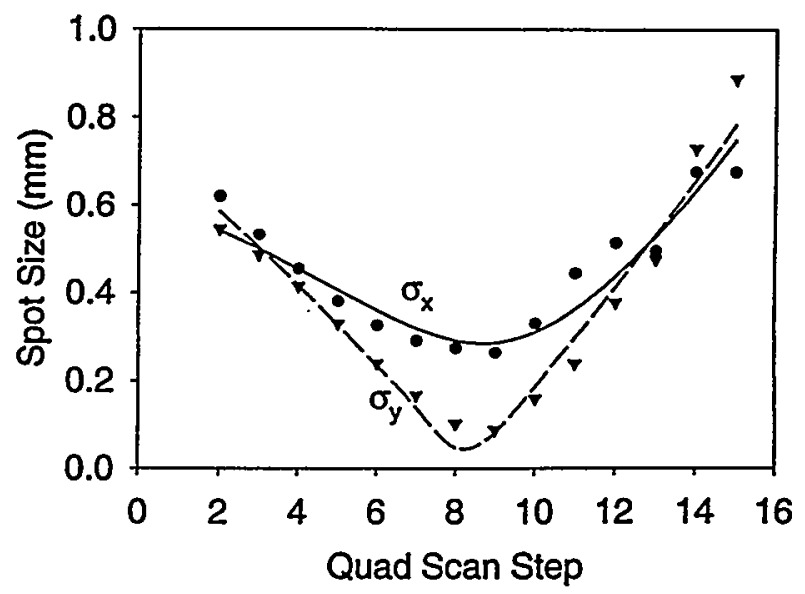

Figure 3: An example of the quadupole field scan versus observed beam sizes: $\sigma_{x}$ (circles) and $\sigma_{y}$ (triangles). Emittances of $\varepsilon_{x, y}<10 \pi \mathrm{mm}$ mrad were determined from the fitted curves. 


\subsection{YAG:Ce Crystal Response Time}

The rf gun's macropulse is typically 30 to $40 \mathrm{~ns}$, shorter than the 80-ns (FWHM) response time of YAG:Ce reported by Graves et al. [10]. This would preclude any submacropulse imaging with this converter. We have used the slow sweep streak module operating at $1-\mu$ s full range to assess the actual response times of our specific crystals. The electron-beam macropulse duration is not a delta function impulse to the system, but it is still sufficiently short that a reasonable measurement is possible. In these data we actually used the DC gun beam with a shortened macropulse of $\sim 20$ ns. As shown in Fig. 4, the measured YAG:Ce response is 105 ns (FWHM). A preliminary assessment of the $1 / \mathrm{e}$ time is also about 112 ns. The streak unit's calibration factor is based on the original factory calibrations.

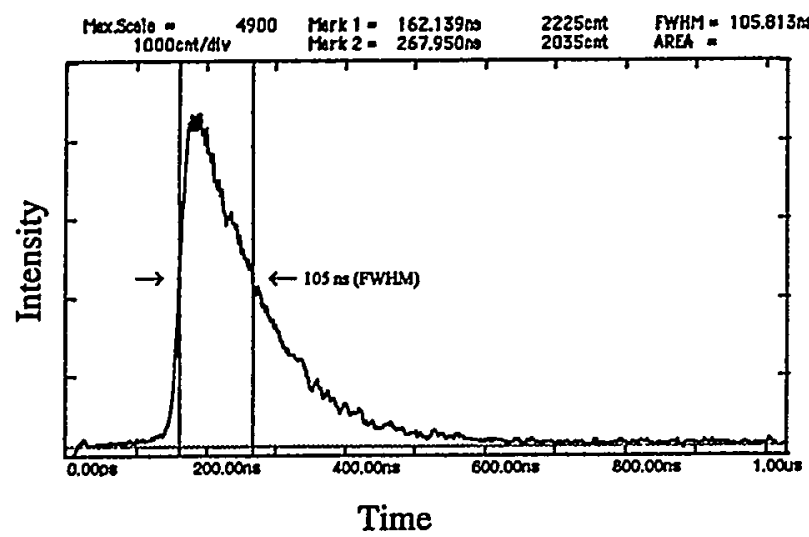

Figure 4: Streak camera data showing the response time of the YAG:Ce crystal to the incident 20-ns electron beam. The effective response is about 105 ns (FWHM). The horizontal axis spans $1 \mu \mathrm{s}$.

\subsection{Bunch Length Measurements}

Assessments of micropulse-averaged bunch length using the synchroscan streak camera have begun. Preliminary results at $540 \mathrm{MeV}$ and for macropulse currents of less than $150 \mathrm{~mA}$ are $3-4 \mathrm{ps}(\sigma)$. Low signal levels required averaging over about 30 macropulses (few seconds) and so may involve some phase slew blurring the image and lengthening the apparent size. Measurements with the optimized gun and an optimized optical path at $220 \mathrm{MeV}$ are planned. Use of a fifth-harmonic cavity signal to optimize bunch length was previously reported by Lewellen et al. [4].

\section{SUMMARY}

In summary, a diagnostics station that can support the characterization of bright electron beams at the end of the APS linac has been tested. It has also been used to attain preliminary data for the rf thermionic gun beam quality at low beam currents. Further experiments are planned this year at high macropulse currents of the if gun and on the PC rf gun.

\section{ACKNOWLEDGEMENTS}

The authors acknowledge M. Borland for adaptation of the on-line emittance software package for this linac case.

\section{REFERENCES}

[1] Proceedings of the Workshop on Fourth Generation Light Sources, M. Cornacchia and H. Winick (Eds.), SLAC, Feb. 24-27, 1997, SERL 92/02 (1997).

[2] Proceedings of the Workshop on Fourth Generation Light Sources, J. L. LaClare (Ed.), ESRF, Grenoble, France (1996).

[3] M. Borland, "An Improved Thermionic Microwave Gun and Emittance Preserving Transport Line," Proc. 1993, PAC, Washington, D.C., May 17-20, 1993, IEEE, Vol. 4, pp. 30153017 (1993).

[4] J. Lewellen et al., "Operation of the APS RF-Gun," Proc. of the 1998 Linac Conf., Chicago, II, Aug. 18-22, 1998 (to be published).

[5] S. G. Biedron et al., "The Operation of the BNLATF Gun-IV Photocathode RF Gun at the Advanced Photon Source," these proceedings.

[6] S. V. Milton et al., Nucl. Inst. and Meth., A407, pp. 210-214 (1998).

[7] A. H. Lumpkin et al., "Optical Techniques for Electron-Beam Characterizations on the APS SASE FEL Project," Proc. of the 1998 FEL Conference, Williamsburg, VA, Aug. 16-21, 1998, NIM (to be published).

[8] A. H. Lumpkin et al., "Time-Resolved Imaging for the APS Linac Beams," Proc. of the 1998 Linac Conference, Chicago, IL, Aug. 18-22, 1998 (to be published).

[9] R. Laird and F. Lenkszus, Argonne National Laboratory, private communication, April 1997.

[10] W. S. Graves, R. D. Johnson, and P. G. O'Shea, "A High Resolution Electron Beam Profile Monitor," Proc. of the 1997 PAC, Vancouver, B.C., May 12-16, 1997, pp. 1993-1995 (1998).

[11] M. Borland, Argonne National Laboratory, OAG Application Programs, Feb. 1999.

[12] elegant code, On-line Users Manual at URL http://www.aps.anl.gov/asd/oag/oagSoftware.shtml 